\title{
Different signals of personality and health from the two sides of the face
}

\author{
Robin S S Kramer, Robert Ward \\ School of Psychology, Bangor University, Bangor, LL57 2AS, Gwynedd, UK; \\ email: psp837@bangor.ac.uk
}

\begin{abstract}
Previous studies demonstrate that people with different personality traits have different looking faces. We investigated whether personality and health information are differently signalled by the two hemifaces. Using composite images created from women with high and low scores on health and personality dimensions, we investigated discrimination accuracy with original and mirrored hemifaces. By comparing discrimination accuracy for particular types of hemiface, we address issues regarding both the location of information signals and how these signals are conveyed. From the hemiface stimuli, participants could accurately identify three of the Big Five traits, along with health. We found differences in which hemiface could be more accurately identified, depending on the expressed trait. Emotional stability and health were more accurately discriminated from the right hemiface, while extraversion showed higher accuracy from the left hemiface. We found evidence for differences between hemifaces related to both directional asymmetries and to other information content. Finally, our results also address ongoing debate about which side of the face is more attractive, as we found attractiveness differences between hemifaces depended upon the personality trait most clearly expressed.
\end{abstract}

\section{Introduction}

We make judgements about people based purely on their facial appearance. While society warns us against such practices, claiming that these perceptions are often unfounded, there is a growing body of evidence suggesting a level of accuracy that may explain why we do so. It is commonly accepted 
that expressions of emotion can be read from both static (Ekman et al 1987) and dynamic (Bassili 1979) properties of the face, although these are transient states that are easily faked (many studies in this area utilise posed expressions, eg Izard 1971). More stable properties of the face are often linked with hormone levels, which have an effect on both morphology and behaviour. For instance, sex hormones like testosterone influence both the shape of the jaw and levels of aggression (Swaddle and Reierson 2002), as well as relative face width and dominance behaviours (Stirrat and Perrett 2010). For women, ovulation affects both facial attractiveness (Penton-Voak et al 1999) and sexual interests and behaviours (Gangestad et al 2002). These effects may be apparent in a study by Boothroyd et al (2008), who showed that indications of sociosexual orientation are accurately perceived from static face images.

There is now growing evidence that shows personality can also be accurately perceived from the static properties of the faces of strangers. Raters are able to identify personality traits from both individual (Kramer et al 2011; Penton-Voak et al 2006; Shevlin et al 2003) and composite (Kramer and Ward 2010; Little and Perrett 2007; Penton-Voak et al 2006) faces with neutral expressions. Composite images are produced by averaging together a group of individual photographs, therefore maintaining the defining characteristics of that group while minimising those characteristics that make each face look individual (Rowland and Perrett 1995). By creating composites based on the 'Big Five' facets of personality, research demonstrates accurate identification of Extraversion, Agreeableness, Conscientiousness, and Neuroticism (Kramer and Ward 2010; Little and Perrett 2007; Penton-Voak et al 2006) for female faces. For male faces, the story is a little less clear, with accurate perception of Extraversion (Little and Perrett 2007; Penton-Voak et al 2006), as well as Agreeableness and Neuroticism (Penton-Voak et al 2006). While people tend to associate attractiveness with possession of more socially desirable traits (Dion et al 1972), and hence facial attractiveness may play some role in accurate perception of these traits, Kramer and Ward (2010) showed that this alone did not provide a sufficient explanatory mechanism for accurate personality 
identification.

There is also some evidence to suggest that the face may signal health, and that this is related to attractiveness (eg Zebrowitz and Rhodes 2004). However, the evidence thus far has remained inconclusive. Identified cues to health include averageness (Rhodes et al 2007), symmetry (Grammar and Thornhill 1994), sexual dimorphism (Perrett et al 1998), skin colour/texture (Stephen et al 2009), and facial adiposity (Coetzee et al 2009). By using composites and controlling for expression, Kramer and Ward (2010) demonstrated that health could be accurately read from the face, even when some of these cues were minimal due to the removal of colour and external facial features. While the use of composites minimises fluctuating asymmetries, a potential cue to health (Weeden and Sabini 2005), it makes any directional asymmetries even more apparent.

Although it appears that certain socially relevant traits are indeed visible in the face, it is still unclear as to what it is about the morphology that makes this possible. While accurate perception has been established with full faces, little has been done to investigate where in the face this information is carried. Kramer and Ward (2010) began to address this issue with the use of 'internal' faces, where the area containing only the eyes, nose, and mouth was visible. By presenting these stimuli in grey-scale, they were able to demonstrate that accurate perception of Agreeableness, Extraversion, Intellect/Imagination, Neuroticism, and physical health was possible without the information provided by skin colouration and external features such as jaw shape. Indeed, in the case of Intellect/Imagination, removal of this information actually improved performance, suggesting that external features may on occasion signal deceptively.

Another way of exploring the location of information in the face involves its laterality. There is no research as yet investigating whether the right and left sides carry identical or differing information with regard to personality and health, and how this may relate to accuracy in signalling. However, there is much evidence demonstrating the existence of differences in perceptions. Note that here and throughout, we will use "left" and "right" to refer to the perspective of the stimulus 
faces, ie the left and right of the actor, not the viewer. It is generally accepted that the left side of the face is more emotionally expressive, even when controlling for side of presentation by utilising mirror-reversed faces (Nicholls et al 2002). In turn, this can lead to perceptions of a more scientific and less emotional person when more of the right side of the face is visible (Lindell and Savill 2010; ten Cate 2002). This effect on observers' perceptions is known by actors, who choose to show more of their left sides when asked to look emotional and more of their right when asked to look scientific (Nicholls et al 1999). Interestingly, the left side of the body during walking is also judged to be more emotionally expressive (Roether et al 2008). There is even evidence that the left side of the face is more emotionally expressive in other species (Fernández-Carriba et al 2002).

Many studies explore these face-side differences by creating chimeras composed of the left side of the face combined with its mirror to produce a symmetrical and realistic face. This method accentuates lateral differences when compared with its right-side counterpart. However, inherent disadvantages of this technique include the duplication of blemishes and the appearance of unnatural lighting/shadows (Kowner 1995). This literature has confirmed that the left side of the face expresses posed emotions more intensely (Sackeim et al 1978) and appears more expressive, even in neutral faces (Zaidel et al 1995). However, there is much disagreement as to whether one side or the other is perceived as healthier (left - Sitton et al 2006; right - Reis and Zaidel 2001) or more attractive (left - Sitton et al 2006; right - Zaidel et al 1995).

We decided to investigate whether the two halves of the face differently signal health and personality, and how these differences might be explained. We measured the accuracy of observers in identifying personality traits from hemiface and mirrored hemiface stimuli. Our design allowed us to make multiple comparisons in order to identify potential differences in the laterality of personality expression in the face. We considered three potential accounts: perceptual bias, information differences between sides, and directional asymmetries. Figure 1 summarises how specific hemiface comparisons allow us to differentiate between accounts. 
(a)

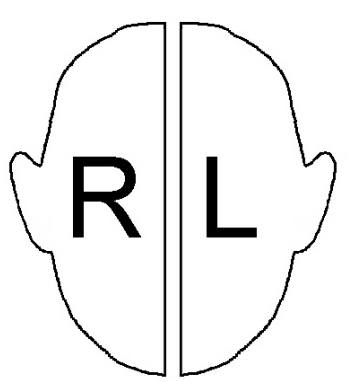

(c)

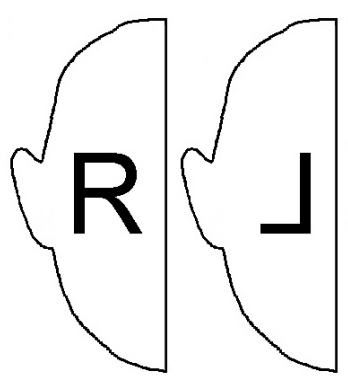

(e)

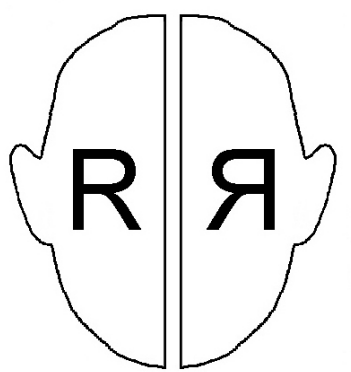

(b)

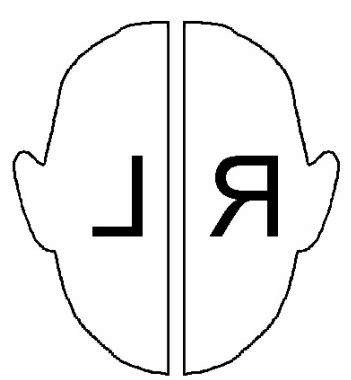

(d)

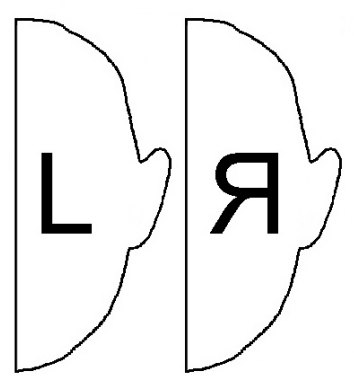

(f)

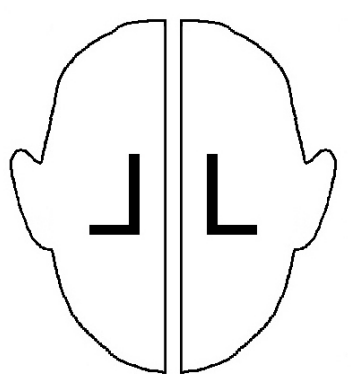

Figure 1. Different hemiface comparisons provide evidence for various accounts of performance difference. (a) Information content and perceptual bias vary; any available directional asymmetry information can be used correctly. (b) Information content and perceptual bias vary, but any directional asymmetries will be misleading. (c,d) Information content varies; some directional asymmetry information can be used correctly. (e,f) Perceptual bias varies but the information content of each hemiface is the same; some directional asymmetry information can be used correctly.

By perceptual bias, we refer to accounts in which more accurate trait judgements from one hemiface over the other reflect biases in the observer's visual processing rather than information in the hemifaces themselves. Although "perceptual bias" in face processing is often used to describe a bias to attend to one side of full faces, a bias could also emerge for hemifaces. For example, it is frequently found that the right hemisphere shows some specialisation for face processing compared to the left hemisphere (Burt and Perrett 1997; Le Grand et al 2003). Specialised face processors might then be biased for face stimuli that would be typically presented in the left hemifield, that is, 
the actor's right hemiface. In principle, such a bias could be found even when hemifaces are centrally presented, if the face invokes an object-based frame of reference for subsequent processing (eg Hommel and Lippa 1995).

A perceptual bias, for the viewer to more readily engage with either a left or a right hemiface, can be easily distinguished from an account in which the two hemifaces actually contain different information. For example, just as the left hemiface may contain more information about emotional state than the right hemiface (Sackeim et al 1978), one hemiface may more accurately signal information about a personality trait.

By directional asymmetries, we refer to one, very specific sort of information difference that produces observer expectations about the information gained from each hemiface. While fluctuating asymmetries randomly deviate from perfect symmetry (eg right hemiface equally likely to be larger or smaller than left hemiface), directional asymmetries show a consistent bias across a population which our perceptual mechanisms have adapted to over time (Rhodes et al 2009). For example, the right hemiface is generally larger than the left hemiface (Farkas and Cheung 1981). Unlike other information differences, the interpretation of directional asymmetries would be disrupted under conditions of mirror reversal.

In the present study, we presented composite hemifaces that reflected real differences between people with different levels of traits. As such, we investigated whether one side of the face produced different accuracy in perceiving these traits in comparison with the other. Composite faces were used in order to more easily investigate population-wide laterality differences. Discrimination accuracy was measured using a two-alternative forced choice methodology.

\section{Method}

\subsection{Participants}

Thirty-two participants ( 25 females; mean age $=20.00$ years, $\mathrm{SD}=3.15$ years $)$ took part for course 
credit. All participants were students in the Psychology programme at Bangor University.

\subsection{Design}

The experiment was defined by three factors describing the stimulus images and presentation: Trait (Agreeableness, Conscientiousness, Extraversion, Emotional Stability, Intellect/Imagination, Health) x Face Type (original or mirrored) x Face Side (left or right of actor). All factors were varied within participants.

\subsection{Stimuli: The composite images}

The face images were adapted from the full-face composite images created by Kramer and Ward (2010). Here we briefly describe how those composites were made, for full details refer to Kramer and Ward (2010). A pool of 63 white European women completed a Big Five personality assessment (Mini-IPIP; Donnellan et al 2006) and health survey (SF-12; Ware et al 1996). Digital photographs of each woman's face were taken, constrained to reflect neutral expression, eyes on the camera; consistent posture, lighting, and distance to the camera; no glasses, jewellery, or make-up; and hair back.

The 15 highest and 15 lowest scorers were identified on each of the Big Five traits (Agreeableness, Extraversion, Conscientiousness, Emotional Stability, and Intellect/Imagination), and Health (based upon the PCS sub-scale of the SF-12). Although the Mini-IPIP specifies Neuroticism, we refer to its reverse, Emotional Stability, so that the high and low scores reflect high and low social desirability for all traits. Separate composite images were made for the high and low scorers using Abrosoft FantaFace Mixer, based on 112 key locations within the face and around the face outline. (All composite images can be seen in Kramer and Ward 2010.) This procedure created composite face pairs reflecting low and high values on each of the six traits. Kramer and Ward (2010) found that the pairs for Extraversion, Agreeableness, Emotional Stability, and Health could 
be accurately identified (all $p$ s $<.001)$.

Hemiface images. Each of the 12 composites taken from Kramer and Ward (2010) was divided in half down the midline, in the midsagittal axes. For each half, a mirror image was also produced (see figure 2), to create 48 hemiface stimuli. By presenting only composite hemifaces, we could explore whether the two sides of the face carried similar or different health and personality information.

(a)

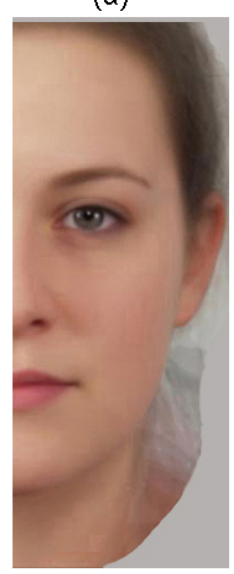

(b)

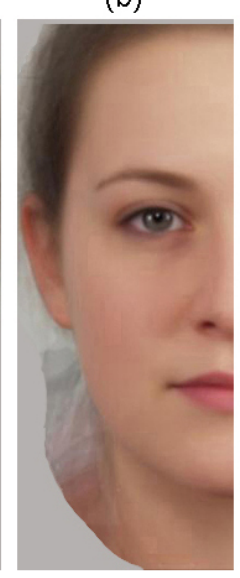

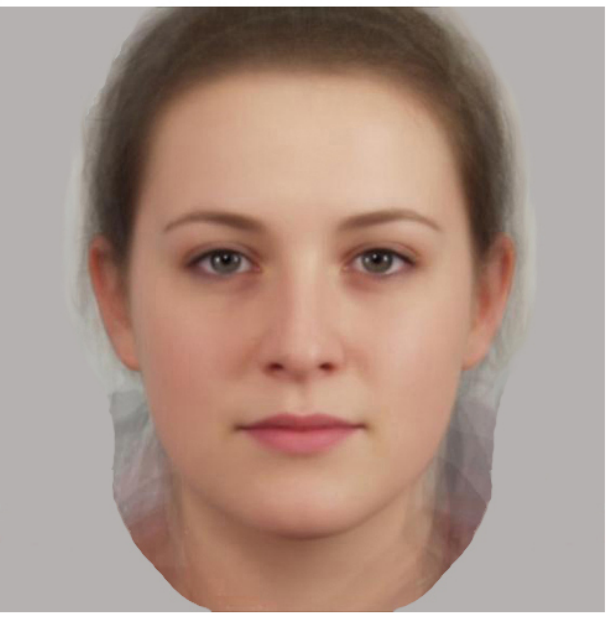

(c)

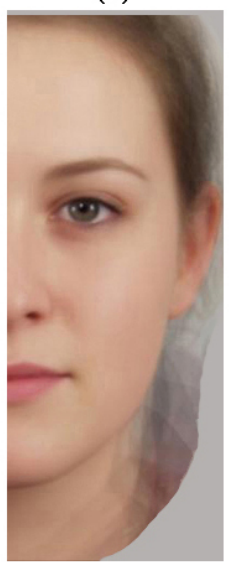

(d)

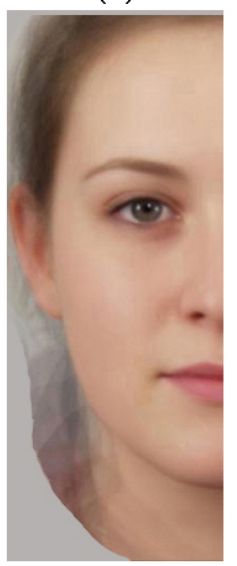

Figure 2. Example stimuli. The full face composite for high extraversion (centre) is cropped to produce (b) the right hemiface and (a) the right hemiface mirrored, along with (c) the left hemiface and (d) the left hemiface mirrored.

\subsection{Procedure}

On each trial, the high and low composite hemifaces were presented to the participant (image size of $175 \times 445$ pixels, or about $5 \times 12$ centimetres on screen), one above and one below the centre of the screen. Viewing distance was not fixed. The task was to judge which hemiface better suited the discrimination statement appearing to the left of the composite pair (see figure 3). Participants indicated their answer by using the mouse to click on the appropriate image, and the next trial then appeared. The experiment was self-paced, and participants were encouraged to make their best answer. 


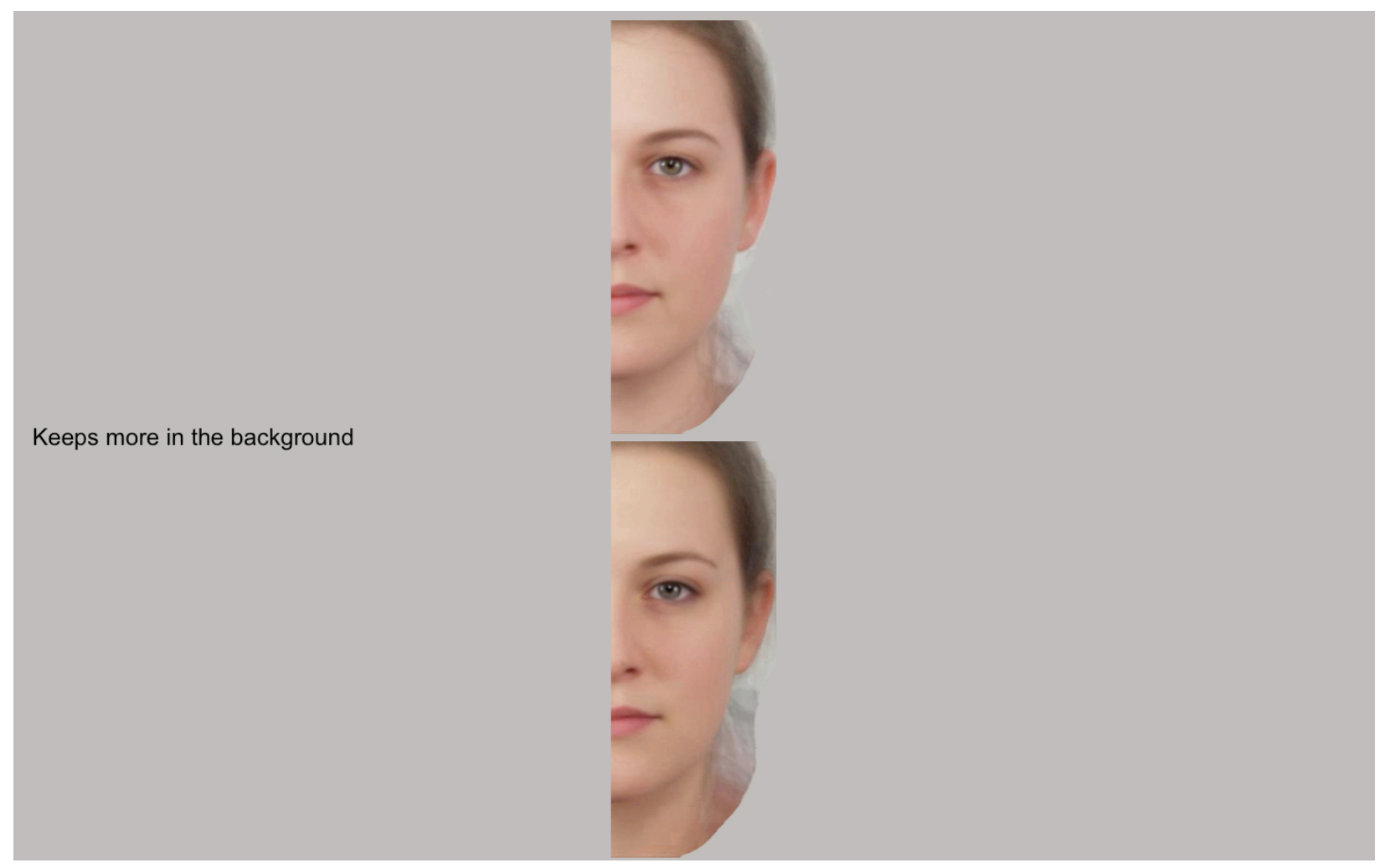

Figure 3. An example stimulus display. Participants clicked on the hemiface that better matched the discrimination statement.

Each of the 24 stimulus pairs was presented four times, each time with a different discrimination statement. For two of the discrimination statements, the correct answer was the lowvalue face, and for two the high-value face. We showed each of these 96 trials twice, counterbalancing the position of the stimuli so that each of the hemifaces would be correctly selected once in the upper and once in the lower screen position. For the Big Five traits, the discrimination statements were taken directly from the four relevant questions of the Mini-IPIP inventory used for scoring the women in the original stimulus creation. For Health, the discrimination statements were taken from four items of the PCS sub-scale of the SF-12. The four items chosen were the ones producing the largest contributions to sub-scale scores for the women in our stimulus pool. We used discrimination statements based on items 1 (health is better), $2 \mathrm{~b}$ (has greater difficulty climbing stairs), 3a (accomplishes less due to health problems), and 5 (pain interferes more with work). All factors were counterbalanced. In addition, the order of trials was 
randomised for each participant.

After completing the discrimination task, participants rated each of the 48 hemifaces for attractiveness. Images were presented one at a time in the centre of the screen, the same size as before, and in random order. Above the image would appear the phrase "How attractive is this face?", and under the image, a written 7-item scale (eg Very Unattractive; Unattractive; Slightly Unattractive; Average; Slightly Attractive; Attractive; Very Attractive). Participants clicked on the appropriate rating with the mouse, and the next image then appeared.

\section{Results}

\subsection{Overall Trait Accuracy}

A 2 (Face Side: left or right) x 6 (Trait: Agreeableness, Conscientiousness, Extraversion, Emotional Stability, Intellect/Imagination, Health) x 2 (Face Type: original or mirrored) ANOVA found a main effect of Trait $\left(F_{5,155}=13.47, p<.001\right)$, demonstrating that accuracy varied for the different traits that were included. We consider these effects in more detail below.

One main interest was in the effects of laterality. Here we found a main effect of Face Side $\left(F_{1,31}=7.22, p=.011\right)$, with accuracy higher for the right side $(M=58.4 \%)$ of the face compared with the left $(M=55.1 \%)$. This was qualified by a Trait $\mathrm{x}$ Face Side interaction $\left(F_{5,155}=6.30, p<\right.$. 001). Figure 4 illustrates how accuracy was significantly higher for the right side of the face compared with the left for Emotional Stability, Intellect/Imagination, and Health (all $p \mathrm{~s}<.04$ ), but lower for Extraversion $(p=.005)$. 


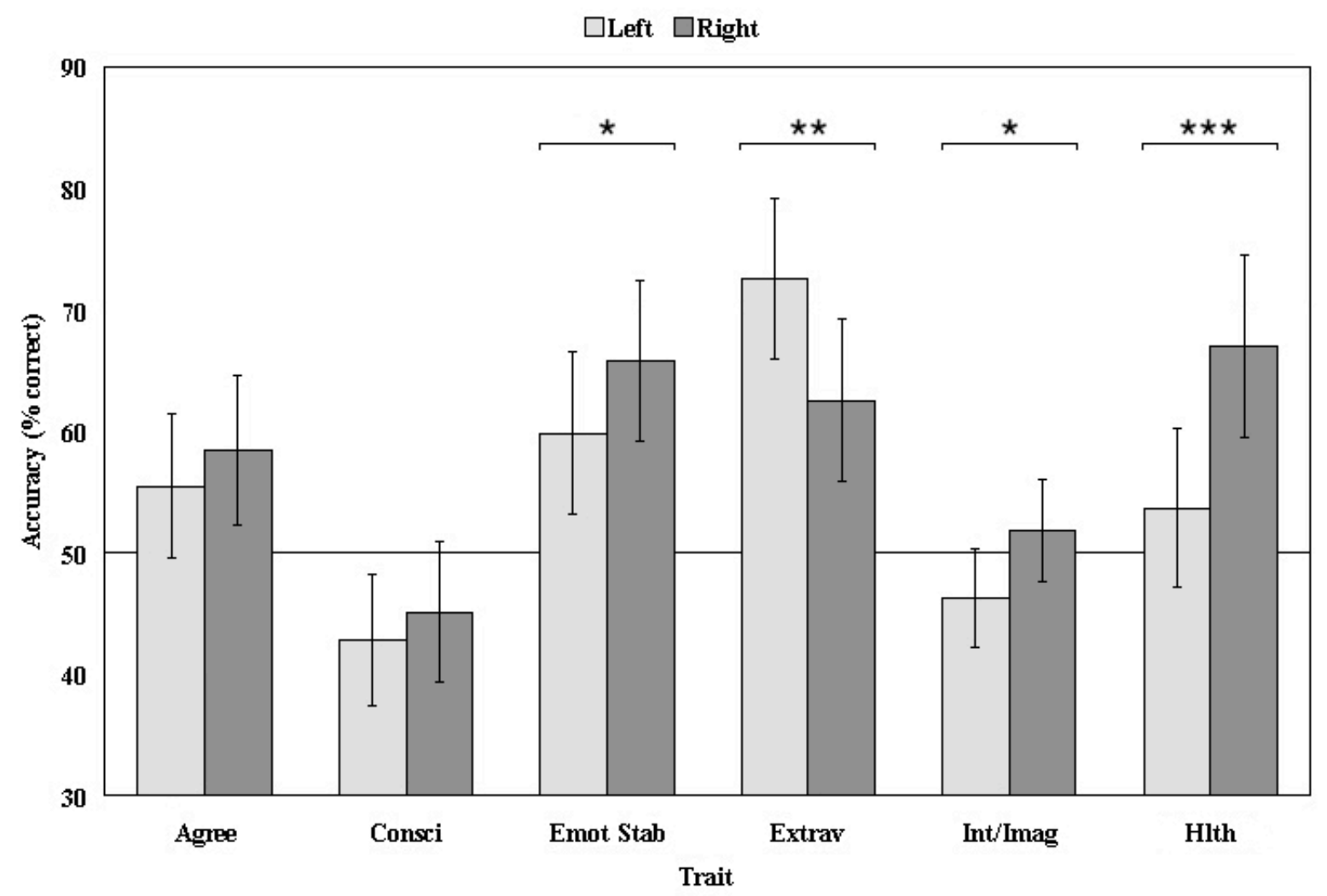

Figure 4. Accuracy on forced-choice (2 alternative) discrimination for the Big 5 personality traits, and for Health, as measured by the PCS sub-scale of the SF-12. Chance performance level indicated by line at 50\%. Error bars indicate $95 \%$ confidence interval and can be used to compare conditions to baseline (ie error bars overlapping the $50 \%$ line are not significantly different from chance). Agree = agreeableness; Consci = conscientiousness; Emot Stab = emotional stability; Extrav = extraversion; Int/Imag = intellect/imagination; Hlth = health. ${ }^{*}$ were significantly different at an uncorrected alpha level of $.05 ; * *$ at $.01 ; * * *$ at .001 .

As figure 4 shows, Extraversion was accurately perceived on both the left $\left(t_{31}=7.03, p<\right.$. $001)$ and right $\left(t_{31}=3.79, p<.001\right)$ sides of the face. Emotional Stability was also accurate in the left $\left(t_{31}=2.97, p=.006\right)$ and right $\left(t_{31}=4.79, p<.001\right)$ sides. Agreeableness performance was above chance for the right side of the face $\left(t_{31}=2.80, p=.009\right)$ and close to significant for the left $\left(t_{31}=1.90, p=.067\right)$. Health was above chance for the right side $\left(t_{31}=4.59, p<.001\right)$.

Conscientiousness was below chance for the left side of the face $\left(t_{31}=2.77, p=.009\right)$. (All $p$ values are uncorrected.)

The results with hemifaces are largely similar to Kramer and Ward's (2010) findings. Using 
full face versions of the stimuli used here, Kramer and Ward found that Extraversion, Agreeableness, Neuroticism (aka Emotional Stability), and Health were identified well above chance levels. In comparison, Conscientiousness was at chance, and Intellect/Imagination was actually identified at slightly below-chance levels.

There was also a main effect of Face Type $\left(F_{1,31}=5.28, p=.029\right)$, with original faces $(M=$ $57.9 \%)$ perceived more accurately than mirrored faces $(M=55.6 \%)$. This was qualified by a Face

Side $\mathrm{x}$ Face Type interaction $\left(F_{1,31}=7.54, p=.010\right)$, where the right side was more accurate than the left for original faces but not mirrored.

No other effects or interactions were significant (all $p s>.269$ ). See Table 1 in the Appendix for all mean accuracy scores separated by Trait, Face Side, and Face Type.

The focus of this experiment is to determine whether accuracy in personality identification is related to differences in the two hemifaces. Given that accuracy scores on both Conscientiousness and Intellect/Imagination were around or below chance levels for both hemifaces, we excluded these traits from the exploration that follows. We would be reluctant to draw any conclusions about mechanisms when hemifaces carried little information that could be accurately perceived anyway.

In the next sections, we look in detail at the predictions from each of three accounts outlined earlier. For the analyses that follow, we did not correct for multiple statistical tests. The comparisons we made were planned in accordance with hypotheses generated from the various accounts that we highlight. It is important to note that the different analyses complemented and supported each other, and as such, made it unlikely that our results were spurious and could have been due to the number of comparisons that were made.

\subsection{Perceptual Bias}

As discussed earlier, a perceptual bias account refers to biases in the observer's visual processing. Although there was an advantage for what appeared to be the right side of the face, as evidenced by 
the interaction of Face Side $x$ Face Type, the perceptual bias account cannot fully explain our results. As described above, we also found a Trait x Face Side interaction, demonstrating that accuracy was not higher with one hemiface for every trait (as a perceptual bias account predicts), and instead differed between traits. Specifically, using original hemifaces (see figure 1a), we found that the original version of the right side of the face was more accurately perceived than the original left side in Health $\left(t_{31}=3.70, p=.001\right)$, and was close to significant in Agreeableness $\left(t_{31}=1.99, p\right.$ $=.056)$ and Emotional Stability $\left(t_{31}=1.85, p=.074\right)$. In contrast, the original left version was more accurate than the original right for Extraversion $\left(t_{31}=2.68, p=.012\right)$. Therefore, the original right hemiface was more accurately perceived for some traits while the original left produced greater accuracy for others.

Similarly, a perceptual bias account failed to explain our results comparing the mirrored hemifaces (see figure 1b). For Health, original right hemifaces produced greater accuracy than original left hemifaces. A perceptual bias account would suggest a bias towards the viewer's left, therefore predicting greater accuracy for the mirrored left hemiface when compared with the mirrored right. However, this was the opposite of what we found, with the mirrored right hemiface producing greater accuracy than the mirrored left for Health $\left(t_{31}=2.94, p=.006\right)$. Likewise for Extraversion, the above results showed that the original left hemiface was more accurate than the original right, suggesting a bias towards the viewer's right side, which in turn predicts greater accuracy for the mirrored right hemiface over the mirrored left. Again our results showed the opposite pattern, with the mirrored left being more accurate than the mirrored right for Extraversion $\left(t_{31}=2.10, p=.044\right)$. Finally, given that original right hemifaces produced close to significant improvements in accuracy over original left hemifaces for Agreeableness and Emotional Stability, we would predict greater accuracy for mirrored right hemifaces over mirrored left hemifaces for these traits if a perceptual bias was apparent. However, we found no differences for these comparisons (all $p \mathrm{~s}>.29$ ). 
In conclusion, no simple explanation of a general perceptual bias can account for this set of results, as observers did not show a consistent bias towards hemifaces that would typically appear in one visual hemifield over the other. Further, using a perceptual bias account to explain specific hemiface advantages led to incorrect predictions with mirrored hemifaces.

\subsection{Information Content}

By comparing an original hemiface to the mirrored version of the other hemiface (see figures 1c and 1d), we can keep any perceptual bias constant, while allowing information content to vary. In addition, directional asymmetry information can be correctly used for the hemiface that is not mirrored. The original right hemiface was more accurate than the mirrored left hemiface in Health $\left(t_{31}=3.22, p=.003\right)$ and Emotional Stability $\left(t_{31}=3.88, p=.001\right)$. In these cases, better performance can be explained by either information content present in the right but not the left hemiface, or by the use of directional asymmetries available in the right hemiface, but unavailable or even misleading in the mirrored left hemiface. However, the mirrored right hemiface led to greater accuracy than the original left hemiface for Health $\left(t_{31}=2.61, p=.014\right)$, and this can only be explained by information content unrelated to directional asymmetries since the mirrored hemiface provided no useful directional asymmetry information.

Similarly, the original left hemiface was more accurate than the mirrored right hemiface in Extraversion $\left(t_{31}=2.40, p=.023\right)$. In this case, better performance can be explained by either information content or the use of directional asymmetries in the left hemiface. However, the mirrored left hemiface led to greater accuracy than the original right hemiface for Extraversion $\left(t_{31}\right.$ $=2.87, p=.007)$, and this can only be explained by information content unrelated to directional asymmetries since the mirrored hemiface provided no valid directional asymmetry information.

\subsection{Directional Asymmetries}


By comparing original and mirrored versions of the same hemiface (see figures 1e and 1f), we keep information other than directional asymmetries constant. Having ruled out perceptual bias as a general explanation, any differences from mirroring can be attributed to the use of directional asymmetries. There were no cases in which the mirrored hemiface produced greater accuracy than the original version of the same hemiface. We found that the original right hemiface was more accurate than the mirrored right hemiface in Agreeableness $\left(t_{31}=2.59, p=.015\right)$ and Emotional Stability $\left(t_{31}=2.52, p=.017\right)$. There was no difference in this comparison for other traits, nor was there any difference for any traits when comparing the original and mirrored left hemifaces.

\subsection{Summarising Accuracy Accounts}

The accuracy results can be summarised with regard to our six traits and three potential accounts as follows. Conscientiousness and Intellect/Imagination were not perceived accurately and so were not pursued further. There was no evidence to support a perceptual bias account for any trait. Both Agreeableness and Emotional Stability showed some evidence of higher accuracy for the right hemiface, and this advantage was best explained by directional asymmetries. Health showed higher right hemiface accuracy while Extraversion demonstrated a left hemiface advantage, and in both cases, evidence supported an account based on differences in information content unrelated to directional asymmetries. These results demonstrate that different traits can be represented in distinct ways between the two sides of the face.

\subsection{Attractiveness}

Previous studies have disagreed about which side of the face is more important for attractiveness judgements. Here we collected attractiveness ratings for different sides of the face reflecting different personality types and different levels of socially desirable traits. We suggest that uncontrolled differences in personality expression might account for some of these previous 
discrepancies.

We averaged together original and mirrored hemiface ratings and then carried out analyses of variance in order to investigate attractiveness differences between the stimuli. A 2 (Composite: high or low) x 2 (Face Side: left or right) x 6 (Trait: Agreeableness, Conscientiousness, Extraversion, Emotional Stability, Intellect/Imagination, Health) ANOVA found a main effect of Composite $\left(F_{1,31}\right.$ $=11.97, p=.002$ ), such that ratings of attractiveness were higher for the more socially desirable composites. There was also a main effect of Trait $\left(F_{5,155}=5.22, p<.001\right)$, illustrating that the composites for different traits were perceived as differently attractive.

While there was no main effect of Face Side $\left(F_{1,31}=0.31, p=.58\right)$, there was a Trait $\mathrm{x}$ Face Side interaction $\left(F_{5,155}=2.73, p=.021\right)$, such that for Conscientiousness, Emotional Stability, Openness, and Health, attractiveness ratings were numerically higher for the left than the right hemiface, while for Agreeableness and Extraversion, the right was numerically higher. This pattern of results demonstrates that whether the left or right side of the face is more attractive can depend upon the trait which is being signalled by the face. There was also a Trait $\mathrm{x}$ Composite interaction $\left(F_{5,155}=15.10, p<.001\right)$. This was produced by differences in composite attractiveness for only certain traits: high Extraversion was rated as more attractive than low $\left(F_{1,31}=43.58, p<.001\right)$, high Health was more attractive than low $\left(F_{1,31}=23.17, p<.001\right)$, and low Intellect/Imagination was more attractive than high $\left(F_{1,31}=15.45, p<.001\right)$. Given these results, any suggestion that one hemiface is more attractive is an oversimplification of a more complicated picture, as with the accuracy results. This may explain why previous studies have failed to agree on this issue.

We also investigated whether attractiveness ratings were associated with discrimination accuracy and socially desirable traits. In particular, we wanted to check whether individual raters might be using an "attractiveness strategy", assigning the face they found more attractive the more socially desirable trait. This strategy cannot produce accurate discrimination unless attractive people actually have (and are not merely perceived to have) more socially desirable traits. Given the 
above-chance levels of accuracy we found for most traits, this account therefore does not seem likely, and attractiveness has not predicted discrimination accuracy with full faces (Kramer and Ward 2010). However, we still thought it important to rule out this strategy if possible. We carried out regression analyses to investigate whether differences in individual participants' ratings of attractiveness for the two composites (high minus low) for each trait and hemiface predicted their accuracy, ie did individual ratings predict subsequent discrimination? Of the six traits for the left hemiface judgements, only extraversion accuracy was significantly predicted by attractiveness ratings $(\beta=.35, p=.049)$. For the right hemifaces, only agreeableness accuracy was predicted by attractiveness ratings $(\beta=-.42, p=.018)$. The negative coefficient means that those who rated the low agreeableness composite as more attractive tended to be more accurate in their judgements for this trait. This is actually in opposition to the "attractiveness strategy" above. However, neither of these results were significant after Bonferroni correction. In line with previous research (Kramer and Ward 2010), attractiveness was unable to satisfactorily explain the accuracy of trait identification.

\section{Discussion}

Previous research has demonstrated accurate perception of certain personality and health traits from static images of the face with neutral expressions (Kramer and Ward 2010; Little and Perrett 2007; Penton-Voak et al 2006). However, little is known about where in the face these signals are carried. The current research demonstrates that the two sides of the face carry different amounts and kinds of information, with Emotional Stability and Health more accurately perceived in the actor's right side compared with the left, while Extraversion showed the opposite pattern. In addition, presenting only half the face still provides enough information for accurate perception of Agreeableness, Extraversion, Emotional Stability, and Health.

In addition, traits differed with regard to how we might best explain their hemiface 
differences in accuracy. Agreeableness and Emotional Stability differences were best explained by directional asymmetries, while Health and Extraversion differences suggested differing information content unrelated to directional asymmetries. By making specific comparisons, we provide a method for the investigation and separation of these accounts.

Our results apply to female faces, and we are unable to speculate how they may generalise to a male population. Previous work has found sex differences for signals from full faces (Little and Perrett 2007; Penton-Voak et al 2006) and this may also be the case for hemifaces. The majority of our observers were also female, and although previous research has not shown that accuracy is affected by observer sex (Little and Perrett 2007), further investigation is needed in order to explore these issues.

The accuracy of trait identification, even from hemifaces, is striking. Our method requires participants to compare two averaged faces, high and low on a trait (see also Kramer and Ward 2010; Little and Perrett 2007). The averaging would be expected to increase signal-to-noise ratio (although it would remove cues from fluctuating asymmetry), and the comparison process surely helps make salient the relatively small differences between the averaged faces that account for the differences in personality. However, even when presenting individual faces, for rating one at a time, correlations between trait values as measured by personality scales and as estimated from images of the full face can be significant (eg Penton-Voak et al 2006). An interesting question for future research is whether the individual hemifaces are similarly revealing.

What might be the basis for the difference between sides in trait expression? Our stimuli contain some low-level differences between sides, as the right side is slightly more in shadow than the left due to the arrangement of lighting when the photographs were taken (see the central, full face in figure 2 for an example). However, small differences in the lighting of left and right sides do not explain our results. First, there is no simple story available that one side of the face contains more information than the other. Instead, our results were marked by an interaction such that 
accuracy was a joint function of the trait being judged and the side of the hemiface presented. Second, mirror reversal affected the accuracy of some traits (Agreeableness, Emotional Stability) even while holding all low-level factors constant. An interesting speculation relates to previous results on the laterality of emotional expressiveness. One explanation could be that the actor's left is more emotionally expressive (Nicholls et al 2002) and hence more affected by transitory aspects of the situation. As such, the right side may be a more temporally stable signal of trait, as opposed to state, information. This might explain why viewers show a bias towards their left visual field when forming impressions (Burt and Perrett 1997), and are more likely to inspect the actor's right hemiface first and for longer periods of time (Butler et al 2005). However, this account would not explain why Extraversion was more accurately perceived in the left hemiface.

Our results also provide some evidence suggesting directional asymmetries with regard to personality expression, likely caused by observer expectations that are affected by mirrored stimuli. For example, if people unconsciously look for certain information in the right side of the face that is not present in the left, then if the right side were mirrored and so appeared to be the actor's left side, observers may treat it differently (ie look for information associated with the left side of the face) and perform worse. While the nature of creating composites will minimise fluctuating asymmetries, directional asymmetries will by definition still be present in our various trait groups. These may be caused by, for example, asymmetrical muscular development in the face sides due to differences in hemispheric specialisation of cognitive activity specific to those traits. Research has already demonstrated group-level asymmetries in face shape and size (eg Farkas and Cheung 1981; Ferrario et al 1995; Simmons et al 2004), with the right side tending to be larger.

Accurate perception of traits from only half the face demonstrates that personality information is present even in this limited stimulus. It also shows accuracy remains when fluctuating asymmetry information is removed. While previous research has shown that fluctuating asymmetry affects judgements of attractiveness (Rhodes et al 1998) and health (Rhodes et al 2001), there is only 
limited evidence suggesting that personality and facial symmetry are related (Fink et al 2005).

Comparison with accuracy on full and internal faces (Kramer and Ward 2010) shows that Extraversion, Emotional Stability, and Health are all signalled from full, internal, and half faces, as is Agreeableness to a more limited extent. As such, although it appears that accuracy decreases when only half the face is presented, much like when only the internal features are shown (Kramer and Ward 2010), these same traits are signalled in all cases. Further investigation might attempt to localise information to even smaller regions, eg the eyes or mouth.

Our attractiveness results found that whether the left or right hemiface is more attractive can depend upon the personality trait signalled by the face. For example, this may explain why previous research has been mixed when exploring this question (Sitton et al 2006; Zaidel et al 1995), as studies in this field have not taken such factors as personality into account during their investigations. How individuals vary along health and personality dimensions may dictate which hemiface is perceived as more attractive. For instance, we have already shown that (accurate) perceptions of health are affected by actual levels on this trait.

In addition, analyses showed that individual perceptions of attractiveness did not account for accuracy in personality judgements. This supports previous research (Kramer and Ward 2010) suggesting people did not base their decisions on perceptions of attractiveness.

In conclusion, we have shown that different personality and health trait signals may be lateralised, and that this in turn affects accuracy in perceptions of these traits. In addition, we have shown that some trait information relates to directional asymmetries in the face, but this account is unable to explain other differences that were found.

\section{References}


Bassili J N, 1979 "Emotion recognition: The role of facial movement and the relative importance of upper and lower areas of the face" Journal of Personality and Social Psychology 372049 2058

Boothroyd L G, Jones B C, Burt D M, DeBruine L M, Perrett D I, 2008 “Facial correlates of sociosexuality" Evolution and Human Behavior 29211 - 218

Burt D M, Perrett D I, 1997 "Perceptual asymmetries in judgements of facial attractiveness, age, gender, speech and expression" Neuropsychologia 35685 - 693

Butler S, Gilchrist I D, Burt D M, Perrett D I, Jones E, Harvey M, 2005 “Are the perceptual biases found in chimeric face processing reflected in eye-movement patterns?" Neuropsychologia 43 $52-59$

Coetzee V, Perrett D I, Stephen I D, 2009 “Facial adiposity: A cue to health?” Perception 381700 1711

Dion K L, Berscheid E, Walster E, 1972 "What is beautiful is good” Journal of Personality and Social Psychology 24285 - 290

Donnellan M B, Oswald F L, Baird B M, Lucas R E, 2006 “The Mini-IPIP scales: Tiny-yeteffective measures of the Big Five factors of personality" Psychological Assessment 18192 203

Ekman P, Friesen W V, O’Sullivan M, Chan A, Diacoyanni-Tarlatzis I, Heider K, Krause R, LeCompte W A, Pitcairn T, Ricci-Bitti P E, Scherer K, Tomita M, Tzavaras A, 1987 "Universals and cultural differences in the judgments of facial expressions of emotion" Journal of Personality and Social Psychology 53712 - 717

Farkas L G, Cheung G, 1981 "Facial asymmetry in healthy North American caucasians" Angle Orthodontist 6170 - 77

Fernández-Carriba S, Loeches A, Morcillo A, Hopkins W D, 2002 "Functional asymmetry of emotions in primates: New findings in chimpanzees" Brain Research Bulletin 57561 - 564 
Ferrario V F, Sforza C, Miani A, Jr, Serrao G, 1995 “A three-dimensional evaluation of human facial asymmetry" Journal of Anatomy 186103 - 110

Fink B, Neave N, Manning J T, Grammer K, 2005 "Facial symmetry and the 'big-five' personality factors" Personality and Individual Differences 39523 - 529

Gangestad S W, Thornhill R, Garver C E, 2002 “Changes in women’s sexual interests and their partners' mate retention tactics across the menstrual cycle: Evidence for shifting conflicts of interest" Proceedings of the Royal Society of London B 269975 - 982

Grammar K, Thornhill R, 1994 "Human (Homo sapiens) facial attractiveness and sexual selection: The role of symmetry and averageness" Journal of Comparative Psychology 108233 - 242 Hommel B, Lippa Y, 1995 “S-R compatibility effects due to context-dependent spatial stimulus coding" Psychonomic Bulletin \& Review 2370 - 374

Izard C E, 1971 The face of emotion (New York: Appleton-Century-Crofts)

Kowner R, 1995 "Laterality in facial expressions and its effect on attributions of emotion and personality: A reconsideration” Neuropsychologia 33539 - 559

Kramer R S S, King J, Ward R, 2011 "Identifying personality from the static, nonexpressive face in humans and chimpanzees: evidence of a shared system for signaling personality" Evolution and Human Behavior 32179 - 185

Kramer R S S, Ward R, 2010 “Internal facial features are signals of personality and health" The Quarterly Journal of Experimental Psychology 632273 - 2287

Le Grand R, Mondloch C J, Maurer D, Brent H P, 2003 “Expert face processing requires visual input to the right hemisphere during infancy" Nature Neuroscience 61108 - 1112

Lindell A K, Savill N J, 2010 "Time to turn the other cheek? The influence of left and right poses on perceptions of academic specialisation" Laterality 15639 - 650

Little A C, Perrett D I, 2007 "Using composite images to assess accuracy in personality attribution to faces” British Journal of Psychology 98111 - 126 
Nicholls M E R, Clode D, Wood S J, Wood A G, 1999 “Laterality of expression in portraiture: Putting your best cheek forward" Proceedings of the Royal Society of London B 2661517 1522

Nicholls M E R, Wolfgang B J, Clode D, Lindell A K, 2002 "The effect of left and right poses on the expression of facial emotion" Neuropsychologia $401662-1665$

Penton-Voak I S, Perrett D I, Castles D L, Kobayashi T, Burt D M, Murray L K, Minamisawa R, 1999 "Menstrual cycle alters face preference” Nature 394884 - 887

Penton-Voak I S, Pound N, Little A C, Perrett D I, 2006 "Personality judgments from natural and composite facial images: More evidence for a "kernel of truth" in social perception" Social Cognition 24490 - 524

Perrett D I, Lee K J, Penton-Voak I, Rowland D, Yoshikawa S, Burt D M, Henzi S P, Castles D L, Akamatsu S, 1998 "Effects of sexual dimorphism on facial attractiveness" Nature 394884 887

Reis V A, Zaidel D W, 2001 "Functional asymmetry in the human face: Perception of health in the left and right sides of the face" Laterality 6225 - 231

Rhodes G, Louw K, Evangelista E, 2009 "Perceptual adaptation to facial asymmetries" Psychonomic Bulletin \& Review 16503 - 508

Rhodes G, Proffitt F, Grady J, Sumich A, 1998 "Facial symmetry and the perception of beauty" Psychonomic Bulletin and Review 5659 - 669

Rhodes G, Yoshikawa S, Palermo R, Simmons L W, Peters M, Lee K, Halberstadt J, Crawford J R, 2007 "Perceived health contributes to the attractiveness of facial symmetry, averageness, and sexual dimorphism” Perception 361244 - 1252

Rhodes G, Zebrowitz L A, Clark A, Kalick S M, Hightower A, McKay R, 2001 “Do facial averageness and symmetry signal health?” Evolution and Human Behavior 2231 - 46

Roether C L, Omlor L, Giese M A, 2008 “Lateral asymmetry of bodily emotion expression” 
Current Biology 18 R329 - R330

Rowland D A, Perrett D I, 1995 "Manipulating facial appearance through shape and color" IEEE Computer Graphics and Applications 1570 - 76

Sackeim H A, Gur R C, Saucy M C, 1978 "Emotions are expressed more intensely on the left side of the face" Science 202434 - 436

Shevlin M, Walker S, Davies M N O, Banyard P, Lewis C A, 2003 “Can you judge a book by its cover? Evidence of self-stranger agreement on personality at zero acquaintance" Personality and Individual Differences 351373 - 1383

Simmons L W, Rhodes G, Peters M, Koehler N, 2004 “Are human preferences for facial symmetry focused on signals of developmental instability?” Behavioral Ecology 15864 - 871

Sitton S C, Waite L, Rivers S C, 2006 "Perception of traits from normal, mirror and chimeric images of faces" American Journal of Psychological Research 214 - 20

Stephen I D, Coetzee V, Law Smith M, Perrett D I, 2009 “Skin blood perfusion and oxygenation colour affect perceived human health" PLoS ONE 4 e5083

Stirrat M, Perrett D I, 2010 "Valid facial cues to cooperation and trust: Male facial width and trustworthiness" Psychological Science 21349 - 354

Swaddle J P, Reierson G W, 2002 “Testosterone increases perceived dominance but not attractiveness of human males" Proceedings of the Royal Society of London B 2692285 2289

ten Cate C, 2002 "Posing as professor: Laterality in posing orientation for portraits of scientists" Journal of Nonverbal Behavior 26175 - 192

Ware J E, Jr, Kosinski M, Keller S D, 1996 “A 12 item short form health survey: Construction of scales and preliminary tests of reliability and validity" Medical Care 34220 - 233

Weeden J, Sabini J, 2005 "Physical attractiveness and health in Western societies: A review" Psychological Bulletin 131635 - 653 
Zaidel D W, Chen A C, German C, 1995 "She is not a beauty even when she smiles: Possible evolutionary basis for a relationship between facial attractiveness and hemispheric specialization" Neuropsychologia 33649 - 655

Zebrowitz L A, Rhodes G, 2004 "Sensitivity to "bad genes" and the anomalous face overgeneralization effect: Cue validity, cue utilization, and accuracy in judging intelligence and health" Journal of Nonverbal Behavior 28167 - 185 


\section{Appendix}

\begin{tabular}{|c|c|c|c|c|c|c|}
\hline \multirow[b]{2}{*}{ Trait } & \multirow[b]{2}{*}{$\begin{array}{l}\text { Face } \\
\text { Side }\end{array}$} & \multirow[b]{2}{*}{ Face Type } & \multirow[b]{2}{*}{ Mean } & \multirow[b]{2}{*}{ Std. Error } & \multicolumn{2}{|c|}{$95 \%$ Confidence Interval } \\
\hline & & & & & $\begin{array}{l}\text { Lower } \\
\text { Bound }\end{array}$ & $\begin{array}{l}\text { Upper } \\
\text { Bound }\end{array}$ \\
\hline \multirow{4}{*}{ Agreeableness } & \multirow{2}{*}{ Left } & Original & 53.1 & 3.8 & 45.4 & 60.9 \\
\hline & & Mirrored & 57.8 & 4 & 49.7 & 66 \\
\hline & \multirow{2}{*}{ Right } & Original & 62.9 & 3.3 & 56.2 & 69.6 \\
\hline & & Mirrored & 53.9 & 3.7 & 46.4 & 61.4 \\
\hline \multirow{4}{*}{ Conscientiousness } & \multirow{2}{*}{ Left } & Original & 43 & 3.1 & 36.7 & 49.2 \\
\hline & & Mirrored & 42.6 & 3.7 & 35.1 & 50.1 \\
\hline & \multirow{2}{*}{ Right } & Original & 47.3 & 3 & 41.2 & 53.3 \\
\hline & & Mirrored & 43 & 4.2 & 34.5 & 51.5 \\
\hline \multirow{4}{*}{ Extraversion } & \multirow{2}{*}{ Left } & Original & 72.3 & 3.6 & 65 & 79.6 \\
\hline & & Mirrored & 72.7 & 3.5 & 65.5 & 79.8 \\
\hline & \multirow{2}{*}{ Right } & Original & 60.5 & 3.6 & 53.3 & 67.8 \\
\hline & & Mirrored & 64.5 & 3.6 & 57 & 71.9 \\
\hline \multirow{4}{*}{ Intellect/Imagination } & \multirow{2}{*}{ Left } & Original & 44.9 & 2.9 & 39 & 50.9 \\
\hline & & Mirrored & 47.7 & 2.7 & 42.2 & 53.1 \\
\hline & \multirow{2}{*}{ Right } & Original & 55.9 & 2.6 & 50.5 & 61.2 \\
\hline & & Mirrored & 47.7 & 3.1 & 41.3 & 54 \\
\hline \multirow{4}{*}{ Emotional Stability } & \multirow{2}{*}{ Left } & Original & 62.5 & 3.8 & 54.7 & 70.3 \\
\hline & & Mirrored & 57 & 4.1 & 48.6 & 65.4 \\
\hline & \multirow{2}{*}{ Right } & Original & 70.7 & 3.5 & 63.6 & 77.8 \\
\hline & & Mirrored & 60.9 & 4.1 & 52.6 & 69.3 \\
\hline \multirow{4}{*}{ Health } & \multirow{2}{*}{ Left } & Original & 54.3 & 3.1 & 48.1 & 60.5 \\
\hline & & Mirrored & 53.1 & 4.3 & 44.3 & 61.9 \\
\hline & \multirow{2}{*}{ Right } & Original & 67.6 & 3.7 & 60.1 & 75.1 \\
\hline & & Mirrored & 66.4 & 4.3 & 57.6 & 75.2 \\
\hline
\end{tabular}

Table 1. Accuracy on forced-choice (two alternative) discrimination for the Big 5 personality traits, and for Health, as measured by the PCS sub-scale of the SF-12. Values are percentages, and chance performance is $50 \%$. Accuracy is illustrated for Trait, Face Side, and Face Type. 